Supporting material for

\title{
Full Spectral Decomposition of Ring Currents
}

E. Steiner ${ }^{\mathrm{a}}$ A. Soncini ${ }^{\mathrm{b}}$ and P.W. Fowler ${ }^{\mathrm{c} *}$

${ }^{a}$ School of Biosciences, University of Exeter, Exeter, EX4 4QD, UK

${ }^{\mathrm{b}}$ Afdeling Kwantumchemie, Departement Chemie, Katholieke Universiteit Leuven, Celestijnenlaan 200F, B 3001 Heverlee, Belgium.

${ }^{\mathrm{c}}$ Department of Chemistry, University of Sheffield, Sheffield, S3 7HF, UK

The material comprises cartesian coordinates (a.u.), total RHF/6-31G** energies and numbers of imaginary frequencies for the (planarity constrained in the cases of cyclooctatetraene and corannulene) optimised structures of the molecules used in the current-density calculations described in the text. 
BENZENE C6H6 (D6H) RHF/6-31G**

$\mathrm{E}=-230.712987$ a.u.

$\mathrm{NIMAG}=0$

$\begin{array}{lllll}\text { C1 } & 2.6362200000 & 0.0000000000 & 0.0000000000\end{array}$

$\begin{array}{lllll}\mathrm{C} 2 & 1.3181100000 & 2.2830334900 & 0.0000000000\end{array}$

$\begin{array}{lllll}\text { C3 } & -1.3181100000 & 2.2830334900 & 0.0000000000\end{array}$

C4 $1.3181100000-2.2830334900 \quad 0.0000000000$

$\begin{array}{llllll}\text { C5 } & -2.6362200000 & 0.0000000000 & 0.0000000000\end{array}$

C6 $-1.3181100000-2.2830334900 \quad 0.0000000000$

H1 $4.6866100000 \quad 0.0000000000 \quad 0.0000000000$

H2 $2.3433050000 \quad 4.0587233176 \quad 0.0000000000$

H3 $\quad-2.3433050000 \quad 4.05872331760 .0000000000$

H4 $2.3433050000-4.05872331760 .0000000000$

H5 $\quad-4.6866100000 \quad 0.0000000000 \quad 0.0000000000$

H6 - $-2.3433050000-4.05872331760 .0000000000$ 
CYCLO-OCTATETRAENE C8H8 (D8H) RHF/6-31G** $\mathrm{E}=-307.517150$ a.u.

$\mathrm{NIMAG}=1$

$\begin{array}{lllll}\text { C1 } & 3.2300273741 & 1.2529847065 & 0.0000000000\end{array}$

$\begin{array}{lllll}\mathrm{C} 2 & -1.2529847065 & 3.2300273741 & 0.0000000000\end{array}$

C3 $3.2300273741-1.25298470650 .0000000000$

C4 $-3.2300273741-1.2529847065 \quad 0.0000000000$

$\begin{array}{llllll}\text { C5 } & 1.2529847065 & 3.2300273741 & 0.0000000000\end{array}$

$\begin{array}{llllll}\text { C6 } & -1.2529847065 & -3.2300273741 & 0.0000000000\end{array}$

$\begin{array}{lllll}\text { C7 } & 1.2529847065 & -3.2300273741 & 0.0000000000\end{array}$

$\begin{array}{lllll}\text { C8 } & -3.2300273741 & 1.2529847065 & 0.0000000000\end{array}$

H1 $5.09199895702 .0784056799 \quad 0.0000000000$

$\begin{array}{lllll}\mathrm{H} 2 & -2.0784056799 & 5.0919989570 & 0.0000000000\end{array}$

H3 $5.0919989570-2.07840567990 .0000000000$

H4 $-5.0919989570-2.07840567990 .0000000000$

H5 2.07840567995 .09199895700 .0000000000

H6 $\quad-2.0784056799-5.0919989570 \quad 0.0000000000$

H7 $2.0784056799-5.0919989570 \quad 0.0000000000$

H8 $\quad-5.0919989570 \quad 2.0784056799 \quad 0.0000000000$

CORONENE C24H12 (D6H) RHF/6-31G** $\mathrm{E}=-915.974077$ a.u.

$\mathrm{NIMAG}=0$

$\begin{array}{lllll}\text { C1 } & 2.6958226241 & 0.0000000000 & 0.0000000000\end{array}$

$\begin{array}{llllll}\mathrm{C} 2 & 1.3479113120 & 2.3346508765 & 0.0000000000\end{array}$

$\begin{array}{lllll}\text { C3 } & -1.3479113120 & 2.3346508765 & 0.0000000000\end{array}$

C4 $1.3479113120-2.3346508765 \quad 0.0000000000$

$\begin{array}{llllll}\text { C5 } & -2.6958226241 & 0.0000000000 & 0.0000000000\end{array}$

C6 $-1.3479113120-2.33465087650 .0000000000$

$\begin{array}{llllll}\text { C7 } & 5.3360948630 & 0.0000000000 & 0.0000000000\end{array}$

C8 $2.6680474315 \quad 4.62119370840 .0000000000$

C9 $-2.6680474315 \quad 4.6211937084 \quad 0.0000000000$

C10 $2.6680474315-4.62119370840 .0000000000$

$\begin{array}{lllll}\mathrm{C} 11 & -5.3360948630 & 0.0000000000 & 0.0000000000\end{array}$

C12 -2.6680474315 -4.6211937084 0.0000000000

$\begin{array}{llllll}\mathrm{C} 13 & 6.6383126006 & 2.3561349290 & 0.0000000000\end{array}$

$\begin{array}{llllll}\mathrm{C} 14 & 1.2786835971 & 6.9270148149 & 0.0000000000\end{array}$

C15 $6.6383126006-2.35613492900 .0000000000$

C16 $-5.3596290036 \quad 4.57087988590 .0000000000$ 
$\begin{array}{lrrr}\text { C17 } & 5.3596290036 & 4.5708798859 & 0.0000000000 \\ \text { C18 } & 1.2786835971 & -6.9270148149 & 0.0000000000 \\ \text { C19 } & -6.6383126006 & -2.3561349290 & 0.0000000000 \\ \text { C20 } & -1.2786835971 & 6.9270148149 & 0.0000000000 \\ \text { C21 } & -5.3596290036 & -4.5708798859 & 0.0000000000 \\ \text { C22 } & 5.3596290036 & -4.5708798859 & 0.0000000000 \\ \text { C23 } & -1.2786835971 & -6.9270148149 & 0.0000000000 \\ \text { C24 } & -6.6383126006 & 2.3561349290 & 0.0000000000 \\ \text { H1 } & 8.6719355541 & 2.3495208880 & 0.0000000000 \\ \text { H2 } & 2.3012230013 & 8.6848769338 & 0.0000000000 \\ \text { H3 } & 8.6719355541 & -2.3495208880 & 0.0000000000 \\ \text { H4 } & -6.3707125528 & 6.3353560458 & 0.0000000000 \\ \text { H5 } & 6.3707125528 & 6.3353560458 & 0.0000000000 \\ \text { H6 } & 2.3012230013 & -8.6848769338 & 0.0000000000 \\ \text { H7 } & -8.6719355541 & -2.3495208880 & 0.0000000000 \\ \text { H8 } & -2.3012230013 & 8.6848769338 & 0.0000000000 \\ \text { H9 } & -6.3707125528 & -6.3353560458 & 0.0000000000 \\ \text { H10 } & 6.3707125528 & -6.3353560458 & 0.0000000000 \\ \text { H11 } & -2.3012230013 & -8.6848769338 & 0.0000000000 \\ \text { H12 } & -8.6719355541 & 2.3495208880 & 0.0000000000\end{array}$ 
CORANNULENE C20H10 (D5H) RHF/6-31G** $\mathrm{E}=-763.192870$ a.u.

NIMAG $=1$

$\begin{array}{lllll}\text { C1 } & 2.2414795841 & 0.0000000000 & 0.0000000000\end{array}$

$\begin{array}{lllll}\mathrm{C} 2 & 0.6926552840 & 2.1317737646 & 0.0000000000\end{array}$

$\begin{array}{lllll}\text { C3 } & -1.8133950761 & 1.3175086428 & 0.0000000000\end{array}$

C4 $0.6926552840-2.13177376460 .0000000000$

C5 $\quad-1.8133950761-1.3175086428 \quad 0.0000000000$

$\begin{array}{lllll}\text { C6 } & 4.7852567449 & 0.0000000000 & 0.0000000000\end{array}$

C7 $\quad 1.4787256566 \quad 4.5510496093 \quad 0.0000000000$

$\begin{array}{llllll}\text { C8 } & -3.8713540290 & 2.8127033431 & 0.0000000000\end{array}$

C9 $1.4787256566-4.5510496093 \quad 0.0000000000$

C10 $-3.8713540290-2.81270334310 .0000000000$

$\begin{array}{llllll}\text { C11 } & 5.7754807218 & 2.5817889990 & 0.0000000000\end{array}$

$\begin{array}{lllll}\text { C12 } & -0.6707055575 & 6.2906252518 & 0.0000000000\end{array}$

C13 5.7754807218 -2.58178899900 .0000000000$

$\begin{array}{llllll}\text { C14 } & -6.1899995528 & 1.3060312171 & 0.0000000000\end{array}$

C15 4.24014894494 .69499189860 .0000000000

C16 - $0.6707055575-6.29062525180 .0000000000$

$\begin{array}{lllll}\text { C17 } & -3.1549245564 & -5.4834535693 & 0.0000000000\end{array}$

$\begin{array}{lllll}\text { C18 } & -3.1549245564 & 5.4834535693 & 0.0000000000\end{array}$

C19 $-6.1899995528-1.30603121710 .0000000000$

C20 4.2401489449-4.6949918986 0.0000000000

H1 $7.78564083732 .8941588152 \quad 0.0000000000$

H2 $\quad-0.3466132696 \quad 8.2989287102 \quad 0.0000000000$

H3 $7.7856408373-2.89415881520 .0000000000$

$\begin{array}{lllll}\text { H4 } & -7.9998596189 & 2.2348611979 & 0.0000000000\end{array}$

H5 $5.1584039312 \quad 6.5102401936 \quad 0.0000000000$

H6 $-0.3466132696-8.29892871020 .0000000000$

H7 $\quad-4.5975718801-6.9177085298 \quad 0.0000000000$

H8 $\quad-4.5975718801 \quad 6.91770852980 .0000000000$

H9 $\quad-7.9998596189-2.23486119790 .0000000000$

H10 $5.1584039312-6.51024019360 .0000000000$ 
BORAZINE N3B3H6 (D3H) RHF/6-31G**

$\mathrm{E}=-241.166859$ a.u.

$\mathrm{NIMAG}=0$

$\begin{array}{lllll}\mathrm{N} 1 & 2.6616299220 & 0.0000000000 & 0.0000000000\end{array}$

$\begin{array}{lllll}\mathrm{N} 2 & -1.3308149610 & 2.3050391280 & 0.0000000000\end{array}$

N3 $-1.3308149610-2.3050391280 \quad 0.0000000000$

$\begin{array}{lllll}\text { B1 } & -2.7264361851 & 0.0000000000 & 0.0000000000\end{array}$

B2 $1.3632180925-2.36116299810 .0000000000$

B3 $\quad \begin{array}{lllll}1.3632180925 & 2.3611629981 & 0.0000000000\end{array}$

H1 $4.54097942520 .0000000000 \quad 0.0000000000$

$\mathrm{H} 2 \quad-2.2704897126 \quad 3.9326035403 \quad 0.0000000000$

H3 $-2.2704897126-3.9326035403 \quad 0.0000000000$

H4 $-4.9797530128 \quad 0.0000000000 \quad 0.0000000000$

H5 $2.4898765064-4.31259261360 .0000000000$

H6 $2.4898765064 \quad 4.31259261360 .0000000000$ 